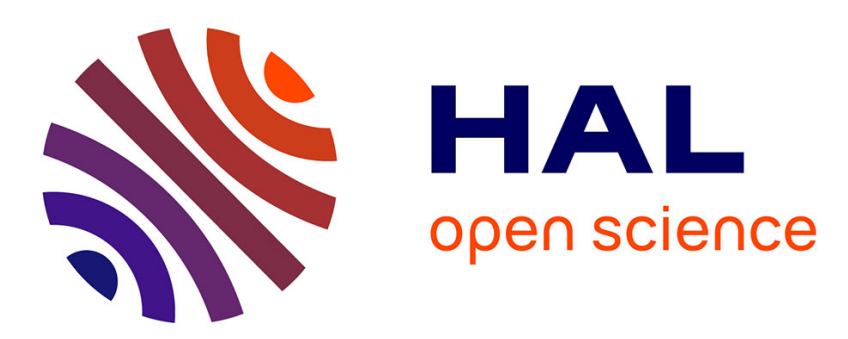

\title{
One-pot synthesis of 5-FU@ZIF-8 and ibuprofen@ZIF-8 nanoparticles
}

Phuoc Hoang Ho, Fabrice Salles, Francesco Di Renzo, Philippe Trens

\section{To cite this version:}

Phuoc Hoang Ho, Fabrice Salles, Francesco Di Renzo, Philippe Trens. One-pot synthesis of 5FU@ZIF-8 and ibuprofen@ZIF-8 nanoparticles. Inorganica Chimica Acta, 2020, 500, pp.119229. 10.1016/j.ica.2019.119229 . hal-02353539

\section{HAL Id: hal-02353539 \\ https://hal.science/hal-02353539}

Submitted on 18 Nov 2020

HAL is a multi-disciplinary open access archive for the deposit and dissemination of scientific research documents, whether they are published or not. The documents may come from teaching and research institutions in France or abroad, or from public or private research centers.
L'archive ouverte pluridisciplinaire HAL, est destinée au dépôt et à la diffusion de documents scientifiques de niveau recherche, publiés ou non, émanant des établissements d'enseignement et de recherche français ou étrangers, des laboratoires publics ou privés. 


\title{
One-pot synthesis of 5-FU@ZIF-8 and ibuprofen@ZIF-8 nanoparticles
}

\author{
Phuoc Hoang Ho, Fabrice Salles, Francesco Di Renzo and Philippe Trens*
}

Ecole Nationale Supérieure de Chimie de Montpellier, Institut Charles Gerhardt des Matériaux, UMR5253 CNRS, ENSCM, UM, 240 Avenue du Professeur Émile Jeanbrau, 34296 MONTPELLIER cedex 5, France.

E-mail: philippe.trens@enscm.fr

\begin{abstract}
In the present study, the synthesis of microporous zinc imidazolate nanoparticles was assessed with and without the presence of ibuprofen or 5-fluorouracil (5-FU) anticancer drug. The drugs loading was ensured by thermogravimetric analysis, nitrogen adsorption/desorption. The crystalline structure of the different prepared materials was monitored by using X-ray powder diffraction. Up to $5 \%$ loading (molar ratio of drug/Zn ${ }^{2+}$ ), there was no evidence of crystalline drugs in the XRD patterns, suggesting an optimized occupation of the ZIF-8 cells. The pattern of the samples remained identical with and without drug. Particle size ranging between 30 and $95 \mathrm{~nm}$ could be deduced according to the Scherrer equation. It was found to depend on the dilution of the reagents, but also on the presence of drugs in the synthesis medium. The morphology of the samples was presented by using scanning and transmission electron microscopies, confirming the size obtained using XRD. The presence of drugs induced a significant increase in the particle size of the synthesized materials; however, those sizes are still in range for drug delivery application (<200 nm). The drugs@ZIF-8 nanoparticles were found to be hexagonal or cubic shaped, depending on the reaction duration.
\end{abstract}

Keywords: Zinc imidazolate; Synthesis; Ibuprofen; Fluorouracil; 5-FU; Encapsulation 


\section{Introduction}

MOFs are a new type of hybrid inorganic - organic materials composed of clusters (metal ions) linked by organic ligands, forming extended network structures. [1] Their intrinsic properties such as remarkable porosity, high thermal/chemical stability and structure tailorability offer a wide range of applications, e.g. gas capture/separation, catalysis, sensors, and healthcare. [2] With such special properties, MOFs are excellent nanomaterials for drug-delivery system in terms of storage, transport, and release of active ingredients or imaging agents. Since Férey and co-workers investigated the first study of iron-based MOFs for biomedical applications, [3] numerous works have been developed for significant extension of MOFs in healthcare. [2,4-7] Due to a mandatory requirement of biocompatible composition, the most common MOFs for applications in biomedicine are based on iron,[3,8-13] or recently Zr.[14] ZIFs, especially ZIF-8 is a special member (based on $\mathrm{Zn}$ ) with specific thermal and chemical stability,[15] and used in various studies for drug delivery [16-18]. For biomedical applications, apart from the aforementioned properties, MOFs must adapt intravenous administration which requires particle size smaller than $200 \mathrm{~nm}$ to avoid embolism.[2] In particular, particle size in range of $1-100 \mathrm{~nm}$ is more preferable due to their unique physical characteristics making them advantageous as drug carriers, therapeutic and deep tissue contrast agents and sensitive probes.[19] Several methods have been used for MOFs preparation including hydro/solvothermal, conventional heating, microwave-assisted, electrochemical, mecanochemical, ionothermal, dry-gel conversion, and microfluidic syntheses. $[13,20]$

Venna et al. studied the crystallinity of ZIF-8 as a function of time in conventional synthesis under magnetic bar stirring at room temperature (RT).[21] They found that the relative crystallinity was achieved after $24 \mathrm{~h}$, and particle size at $1 \mathrm{~h}$ was around $250 \mathrm{~nm}$ while the size was doubled after $12 \mathrm{~h}$. The surface area was around $800 \mathrm{~m}^{2} \mathrm{~g}^{-1}$ at $12 \mathrm{~h}$. Zhuang et al. reported that uniform $70 \mathrm{~nm}$ particles with single-crystalline structure of fluorescein-encapsulated ZIF-8 can be obtained after 5 min of solvothermal synthesis in methanol at RT under stirring of magnetic bar.[22] In another work, Cravillon and coworkers controlled the nano- and microcrystal formation of ZIF-8 by modulators such as sodium formate, 1-methylimidazole, and n-butylamine using conventional stirring at room 
temperature for 24 h.[23] A particle size of around $65 \mathrm{~nm}$ was observed in absence modulator, while it could go down to $10 \mathrm{~nm}$ in presence of n-butylamine. They also reported that stirring resulted in a wider range of particle size distribution compared to static conditions.

Other synthesis methods are important in order to control particle size, size distribution as well as morphology, all of which are crucial features of porous materials for catalytic reactions, adsorption and separation of molecules. Furthermore, nanocrystalline MOFs with high adsorption capacities are envisioned for biomedical applications. For these targets sonochemical and microwave-assisted (MW) methods have been applied.[20] $\mathrm{MW}$-assisted method allows a direct interaction of the radiation with the solution/reactants and hence results in a very energy efficient method of heating. It is widely used for MOFs syntheses mainly focusing on the acceleration of the crystallization, the formation of nanoscale products, as well as improvement of product selectivity and purity.

In sonochemical syntheses, the wavelength of ultrasound is much larger than molecular dimensions and thus there is no direct interaction between ultrasound and molecules responding for chemical reactions. However, high-energy ultrasound interacts with liquid resulting in the cyclic formation of tiny bubbles, growth, and collapse, which is so-called cavitation. The formation of cavitation leads to rapid release of energy with heating and cooling rate of $>10^{10} \mathrm{~K} \mathrm{~s}^{-1}$, temperature of $\sim 5000 \mathrm{~K}$, and pressure of $\sim 1000$ bar. In homogeneous liquid, chemical reactions can take place within cavity (extreme conditions) at the interface (intermediate temperature and pressure) or in the bulk media, where intense shear forces are present.[20] Sonochemistry was applied to the investigation of MOFs starting in 2008 but it is still largely unexplored. Compared to conventional heating, sonochemical synthesis not only can promote the formation of nucleation but also can help dispersing the nuclei in a homogeneous fashion.[20]

In this study, we focused on the optimization of physicochemical parameters allowing for the synthesis of crystalline ZIF-8 nanoparticles. We varied the solution concentration, the molar ligand/metal ratio, the synthesis time and temperature as well as type of solvent. In a second step, we demonstrated that ibuprofen or 5-FU could be included in the cavities of ZIF-8 via one-pot synthesis. 


\section{Experimental}

\section{1. Preparation of ZIF-8 and encapsulation of drugs in ZIF-8 nanoparticles}

ZIF-8 materials were mostly prepared by sonochemical method, in which four parameters were studied including synthesis time, temperature, concentration of precursors, and type of solvents. In a typical synthesis, each precursor, $0.94 \mathrm{~g}$ of zinc nitrate hexahydrate $\left(\mathrm{Zn}\left(\mathrm{NO}_{3}\right)_{2} .6 \mathrm{H}_{2} \mathrm{O}, 98 \%\right.$, Sigma-Aldrich) and $3.3 \mathrm{~g}$ of 2-methylimidazole (99\%, SigmaAldrich), was dissolved separately in a beaker containing $20 \mathrm{~mL}$ of methanol $(\mathrm{MeOH})$ and stirred for 15 min until it was completely soluble. The two beakers were then placed in water bath $\left(30^{\circ} \mathrm{C}\right)$ of an ultrasound instrument (FB15047, Fisher Scientific, ultrasound frequency $37 \mathrm{kHz}$, power consumption total $90 \mathrm{~W}$ ) for $5 \mathrm{~min}$. The 2-methylimidazole ligand solution was then quickly added to the zinc nitrate solution and the ultrasound was turned on for $60 \mathrm{~min}$. Noted that the molar ratio of $\mathrm{MeOH} / \mathrm{Zn}$ after mixing the two solutions was 333. The milky solution was centrifugated at 10,000 rpm (Centrifuge 5804, Eppendorf) and washed thrice with $40 \mathrm{~mL}$ of methanol (100\%, VWR Chemicals) for each. After washing the ZIF-8 product was dried at $80{ }^{\circ} \mathrm{C}$ for $12 \mathrm{~h}$. For comparison purpose, one sample was prepared by the same condition but using conventional stirring with a magnetic bar for $2 \mathrm{~h}$ at $30^{\circ} \mathrm{C}$, . Using methanol solvent, the synthesis time was varied for $5,10,15,25,60$, and 120 min while the volume of methanol used was also changed to 40,60 , and $80 \mathrm{~mL}$ resulted in eventual $\mathrm{MeOH} / \mathrm{Zn}=666,999$, and 1332 , respectively. In addition, the syntheses were performed in other solvents including water, ethanol, 1propanol, and DMF. Volume of $40 \mathrm{~mL}$ solvent was used to dissolve each precursor, Zinc nitrate or 2-methyl imidazole and the synthesis was carried out at $60 \mathrm{~min}$.

Encapsulation of Ibuprofen (IBU) or 5-Fluorouracil (5-FU) in ZIF-8 structure was performed by one-pot synthesis in which a given amount of drug (Ibuprofen 98\%, SigmaAldrich) or 5-Fluorouracil, 99\%, Sigma-Aldrich) was added together with 2methylimidazole when preparing the solution of organic ligand. Molar ratio of drug per $\mathrm{Zn}^{2+}$ were 2.5 or $5 \%$ for IBU and $5 \%$ for 5 -FU and the samples were denoted $2.5 \%$ IBU@ZIF-8, 5\% IBU@ZIF-8, and 5\% 5-FU@ZIF-8, respectively.

\subsection{Characterization of the materials}


X-ray diffraction (XRD) measurement was conducted using D8 Advance Diffractometer (Bruker AXS, Germany, CuK $\alpha$ radiation and a Ni filter). The patterns were recorded in $2 \theta$ range of $2-40^{\circ}$ with an angular step size of $0.0105^{\circ}$ and counting time per step was $1 \mathrm{~s}$. Particle size and cell parameter were average values calculated for the first five peaks of XRD pattern, using equation (1) and (2), respectively.

$$
\begin{aligned}
\tau & =\frac{0.9 \lambda}{\beta \cos \theta} \\
a & =d_{h k l} \sqrt{h^{2}+k^{2}+l^{2}} \text { (Cubic structure) } \\
d & =\frac{\lambda}{2 \sin \theta}
\end{aligned}
$$

Where $\tau$ is mean size, $\lambda$ is $X$-ray wave-length, $\beta$ is the full width half maximum intensity (FWHM), and $\theta$ is the Bragg angle, hkl are Miller indices of planes.

Morphology of ZIF-8 was analyzed by scanning electron microscope (SEM) and transmission electron microscope (TEM) using a Hitachi S2600N microscope (Hitachi, Japan) JEOL 1200 EX2 microscope (Hitachi, Japan), respectively. For TEM analysis, fine powder of materials was suspended in ethanol and the suspension was then dropped wise on a $\mathrm{Cu}$ grid and dried at $100^{\circ} \mathrm{C}$ before doing the analyses. In both cases of SEM and TEM, the particle size distributions were determined by ImageJ software with data measured over 150 particles.

Textural properties of ZIF-8 materials were determined by nitrogen adsorption and desorption at $-196{ }^{\circ} \mathrm{C}$ using Micromeritics Tristar instrument. Prior to analysis, $50 \mathrm{mg}$ of sample was degassed at $100^{\circ} \mathrm{C}$ for $8 \mathrm{~h}$. The specific surface area was calculated using BET model in relative pressure range of $0.01-0.1 \mathrm{p} / \mathrm{p}^{\circ}$ while the pore volume and the external surface area were assessed using $t$-plot method.

TGA Thermal gravimetric measurements were analyzed using a TA Instruments SDT Q600 Thermal Gravimetric Analyzer. Approximately $10 \mathrm{mg}$ of the sample was used for each analysis. The experiment was performed in temperature range from 40 to $900^{\circ} \mathrm{C}$ with a ramping rate $10^{\circ} \mathrm{C} \mathrm{min}-1$ under $60 \mathrm{~mL} \mathrm{~min}^{-1}$ of $\mathrm{N}_{2}$. 


\section{Results and Discussion}

The synthesis conditions of ZIF-8 were optimized based on the literature as there are still issues on the best conditions required producing high yields of ZIF-8 with optimum textural and morphological properties.[24,25] Even though the synthesis conditions could be modified upon the addition of drugs in the synthesis medium, we first focused on the synthesis of pure ZIF-8. We first assessed the influence of the experimental conditions, more precisely, stirring, reagents concentration, and reaction medium and time. In Figure 1 , the XRD patterns of synthesized ZIF-8 are compared in the case of magnetic and ultrasound stirring for two hours of reaction.

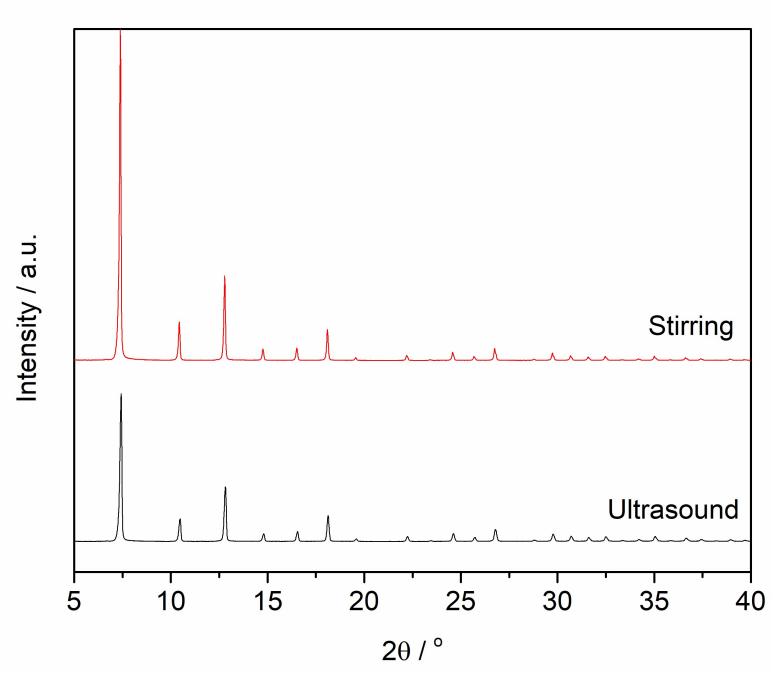

Figure 1. XRD patterns of ZIF-8 nanoparticles prepared by (top) magnetic stirring, (bottom) ultrasound medium.

The diffractograms look very similar, with a good accordance with former published XRD patterns for ZIF-8. [26] The morphology of the obtained nanoparticles is also very similar, as seen in Figure 2. The particle size is narrow in both cases, which suggest homogeneous reaction conditions. It can be deduced that, when possible, ultrasound method should be preferred, due to a shorter time of reaction. We therefore decided to only focus on ultrasound synthesis reactions optimization. 

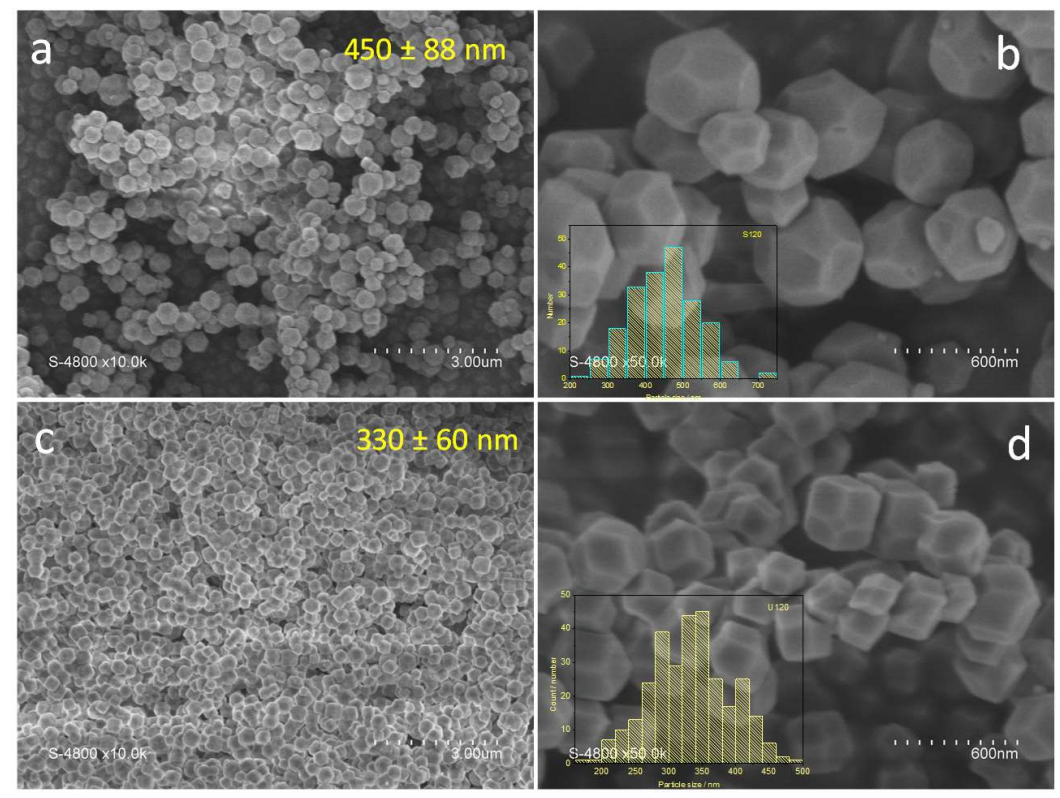

Figure 2. SEM images of the materials prepared using (top) magnetic stirring, (bottom) ultrasound medium. (Right) Higher magnification of Figures 2a and 2c.

Additionally, it must be noted that ZIF-8 can also be synthesized by mechanical synthesis.[27] However, ball milling induced irreversible trapping of iodine species which is not the topic of our study. Lee et al. prepared ZIF-8 by six different methods including solvothermal, microwave-assisted, sonochemical, mechanochemical, dry-gel and microfluidic method.[24] When comparing the catalytic activity of the ZIF-8 for the Knoevengel condensation reaction, they found that the catalysts prepared by dry-gel and sonochemical routes exhibited superior performance due to their significantly smaller particle sizes than those of other methods. However, the catalyst prepared by the sonochemical route in that study showed a particle size of $300-500 \mathrm{~nm}$, which was probably not suitable for medicine applications.

\subsection{Synthesis time for ultrasound method}

Ultrasound syntheses were performed using different reaction duration, up to two hours. The obtained materials were characterized by powder X-ray diffraction. The results are presented in Figure 3. 


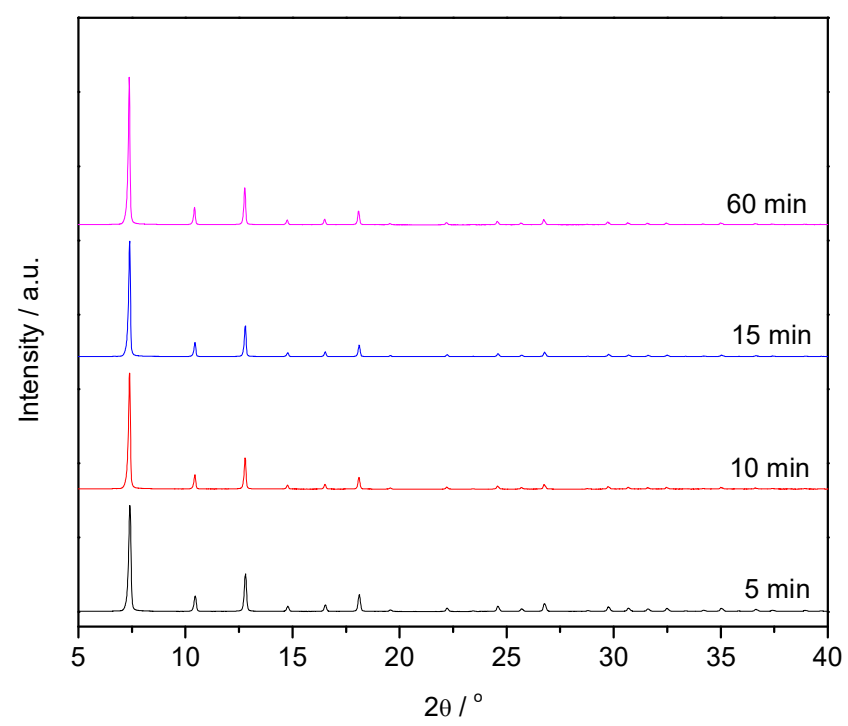

Figure 3. XRD patterns of ZIF-8 nanoparticles prepared using different reaction times.

It can be seen that after 5 minutes, ZIF-8 has already formed, as the usual XRD pattern of ZIF-8 is displayed.[26] Increasing the reaction duration leads to more intensive diffraction peaks but narrower full width half maximum (FMHW), suggesting larger particle size. However, we focused on a reaction time of 60 minutes due to a plateau in yield at this duration $(\sim 30 \%)$. Increasing the reaction time allows for the production of larger nanoparticles which are less suitable for drug release applications. Indeed, in Figure 4, the SEM pictures of the materials prepared with increasing reaction times are shown. Whereas the particle size averages $240 \mathrm{~nm}$ when the reaction time is set to 10 minutes, it increases up to $330 \mathrm{~nm}$ for 120 minutes of reaction duration (Figure 2d). 

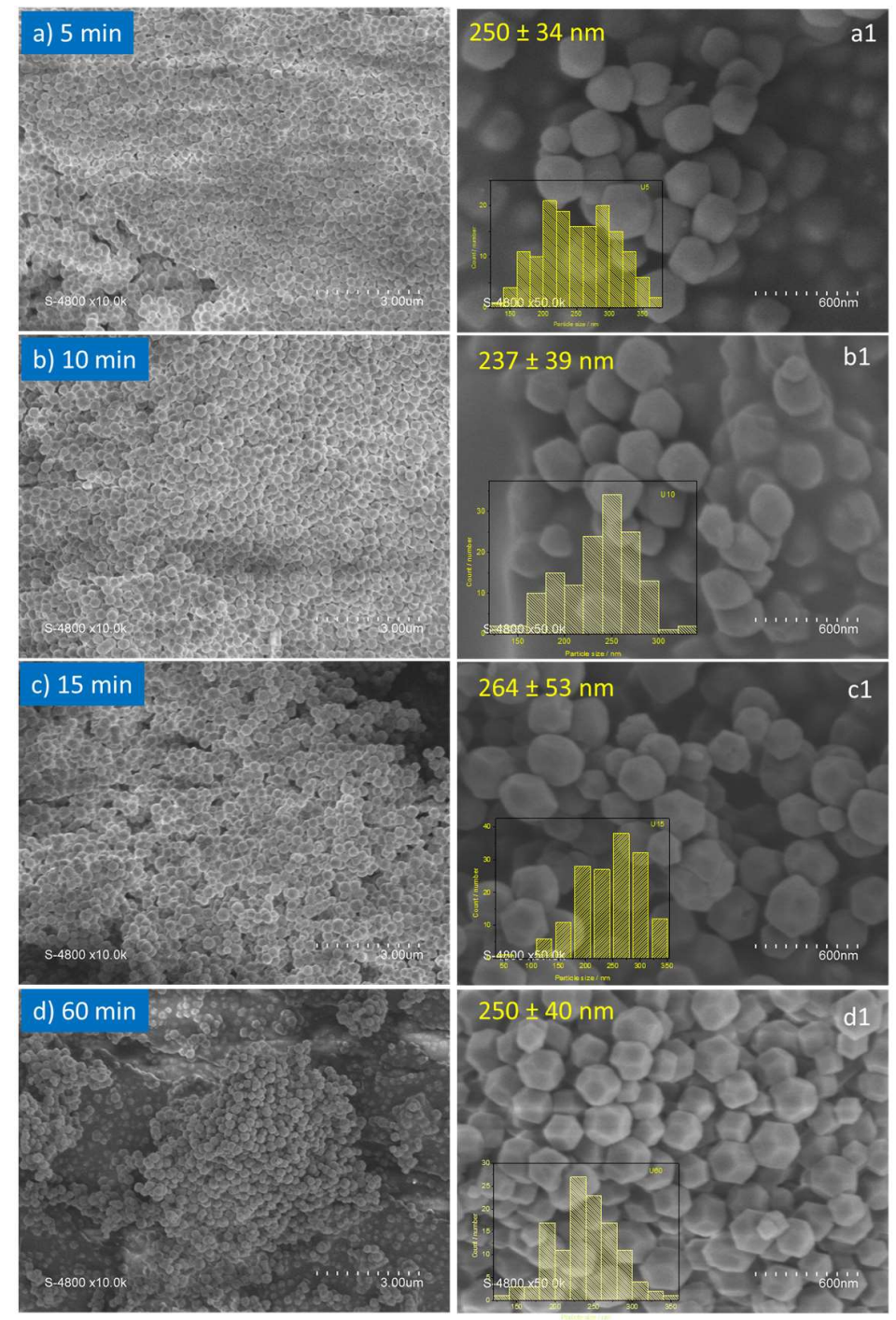

Figure 4. Scanning Electron Microscopy of ZIF-8 nanoparticles prepared with different synthesis duration at increasing magnification from left to right.

However, these sizes are still too large as compared to the optimal particle size for in vivo drug release, typically around $150 \mathrm{~nm}$. We therefore studied the influence of the reagents dilution on the particle size distribution. In Figure $5 \mathrm{a}$, we reported the XRD of the ZIF-8 nanoparticles obtained using different dilutions ratios.

\subsection{Influence of dilution on the ZIF-8 particle size.}

Increasing the dilution, through the methanol/Zn molar ratio, leads to a decrease of the height of the diffraction peaks. Indeed, at low dilution (methanol/Zn $=333$ ), the obtained 
diffractogram shows intense peaks, compared to higher dilutions. It would seem wise to choose this particular dilution; however, the yield increases when the dilution factor increases as shown in Figure $5 b$.
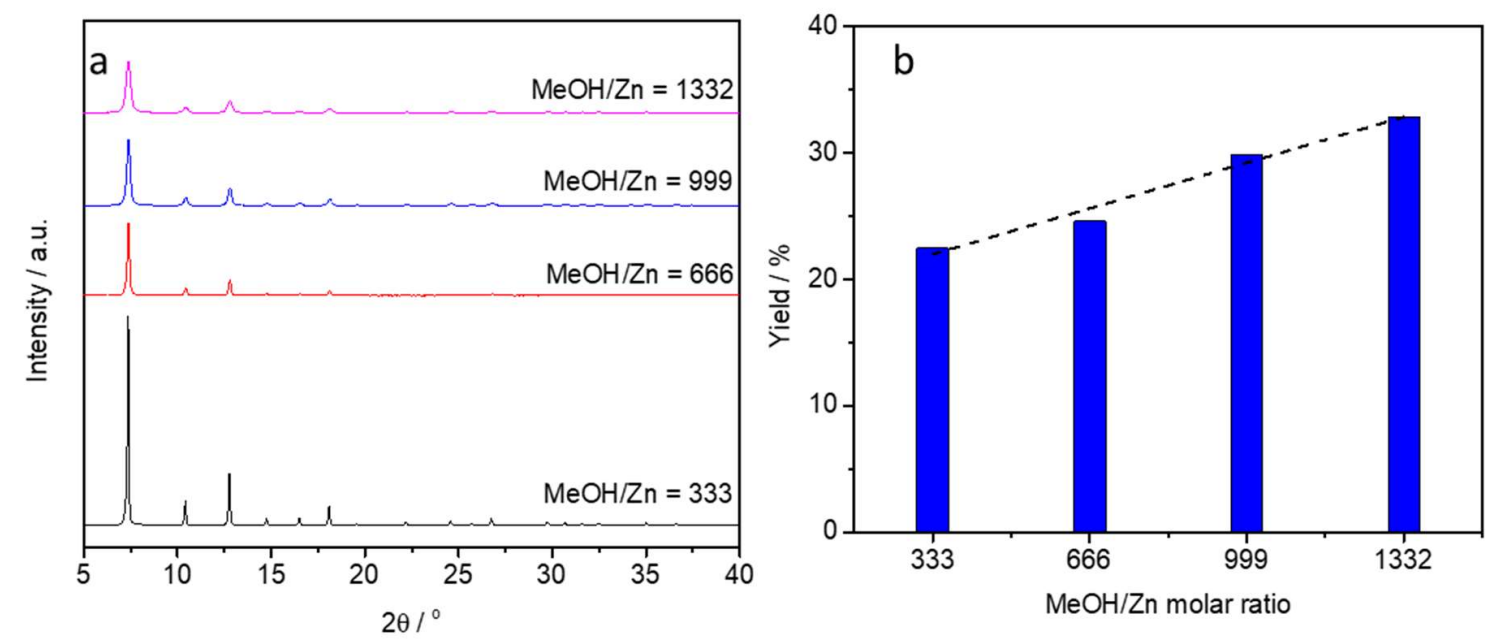

Figure 5. (a) XRD patterns for ZIF-8 nanoparticles using different dilution ratios; (b) Yield versus reagents dilution factor.

Electron microscopies reveal the occurrence of hexagonal or cubic-shaped nanoparticles differing in size, according to the dilution factor (Figure 6). 


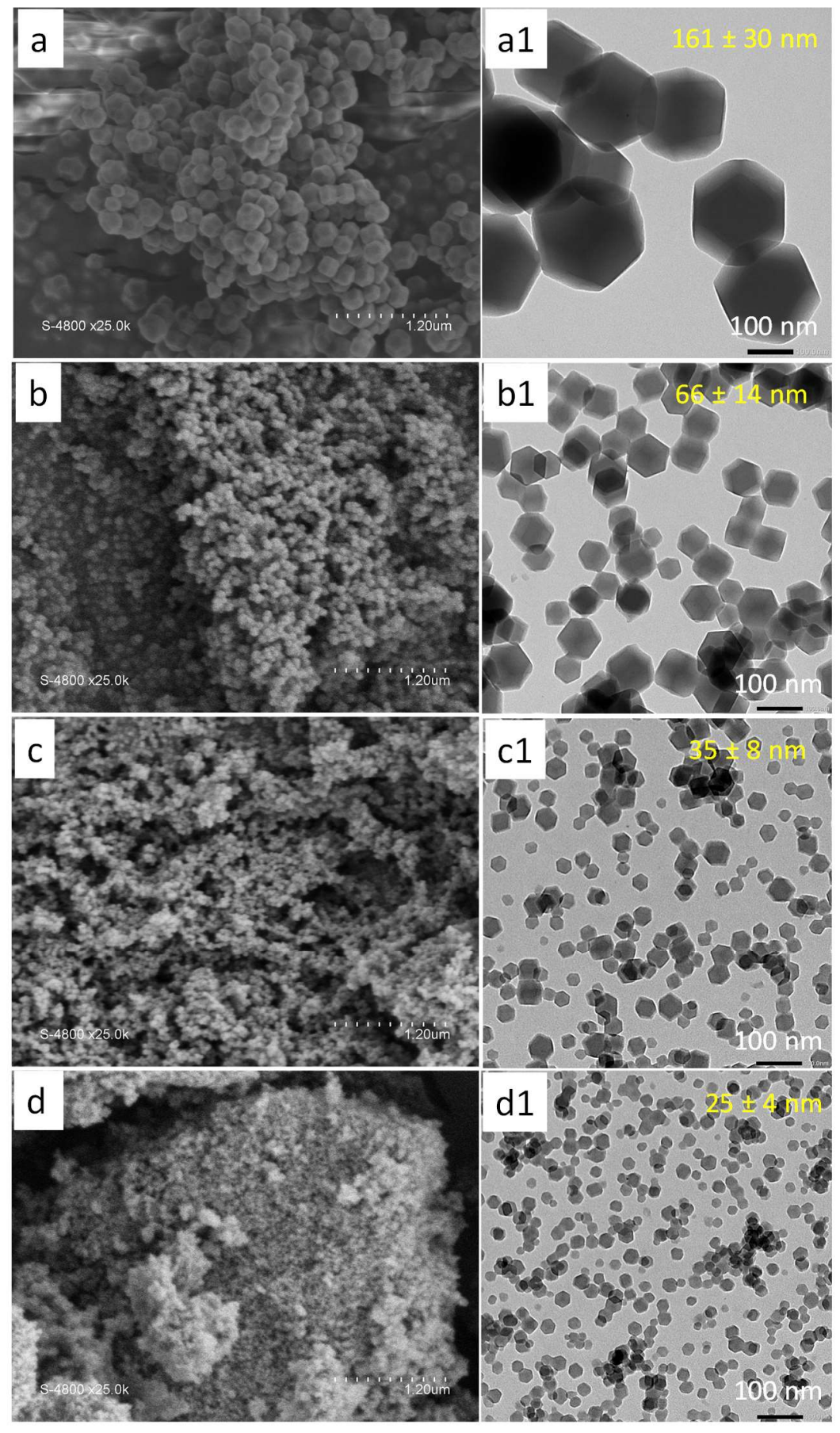

Figure 6. (left) Scanning and (right) transmission electron microscopy of ZIF-8 nanoparticles for different dilution ratios. (a) Dilution ratio $=333$; (b) Dilution ratio $=666$; (c) Dilution ratio $=999 ;$ (d) Dilution ratio $=1332$. 
This trend was confirmed by plotting the average particle size obtained using the Sherrer equation on the XRD patterns of Figure 5 versus the dilution ratio (Figure 7). These consistent results confirm the influence of dilution to provide small ZIF-8 nanoparticles.

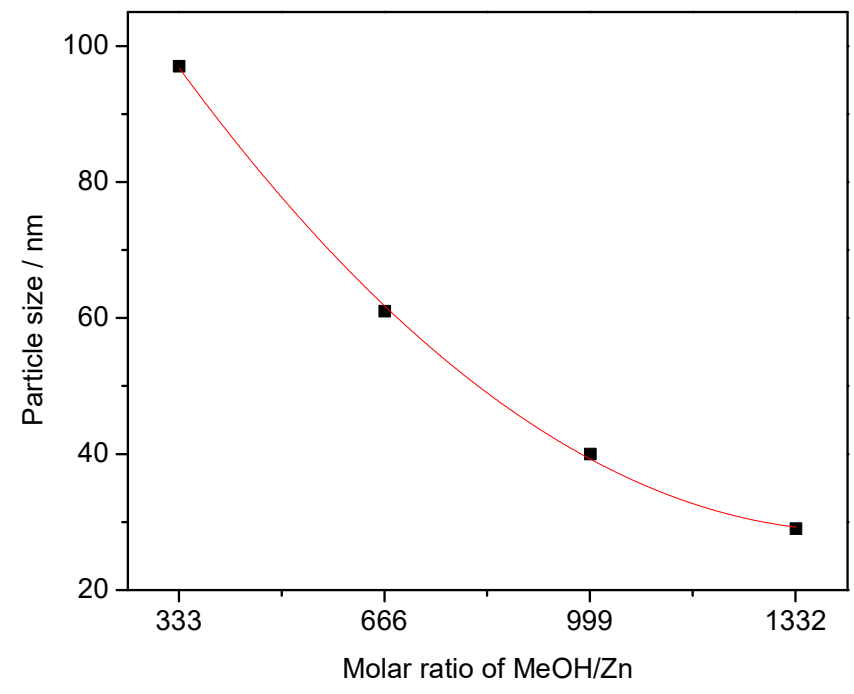

Figure 7. Average particle size obtained using the Scherrer equation applied on Figure 5 versus the dilution factor.

Additionally, we performed nitrogen adsorption on these materials to reveal the differences in terms of textural properties. As the particle size is modified, it can be expected to notice differences in terms of external surface extent and interparticular porosity. The corresponding sorption isotherms are presented in Figure 8. They exhibit a similar shape, typically, a type I sorption isotherm, attributed to microporous materials, as usually found for ZIF-8 materials. The specific surface areas, derived using the t-plot method yielded similar results confirming that the size of the nanoparticles had little influence on the specific surface area (between 1680 and $1780 \mathrm{~m}^{2} . \mathrm{g}^{-1}$ ). It can be deduced that most the surface is located in the microporosity of the materials, rather than developed in the external surface, despite the fact that the particles are in the nanoscale range.

A difference can be noticed between these sorption isotherms, which concerns the high relative pressure range. As the dilution factor increases, a hysteresis loop develops and become very large for the highest dilution factor used (methanol/Zn ratio $=1332$ ). 


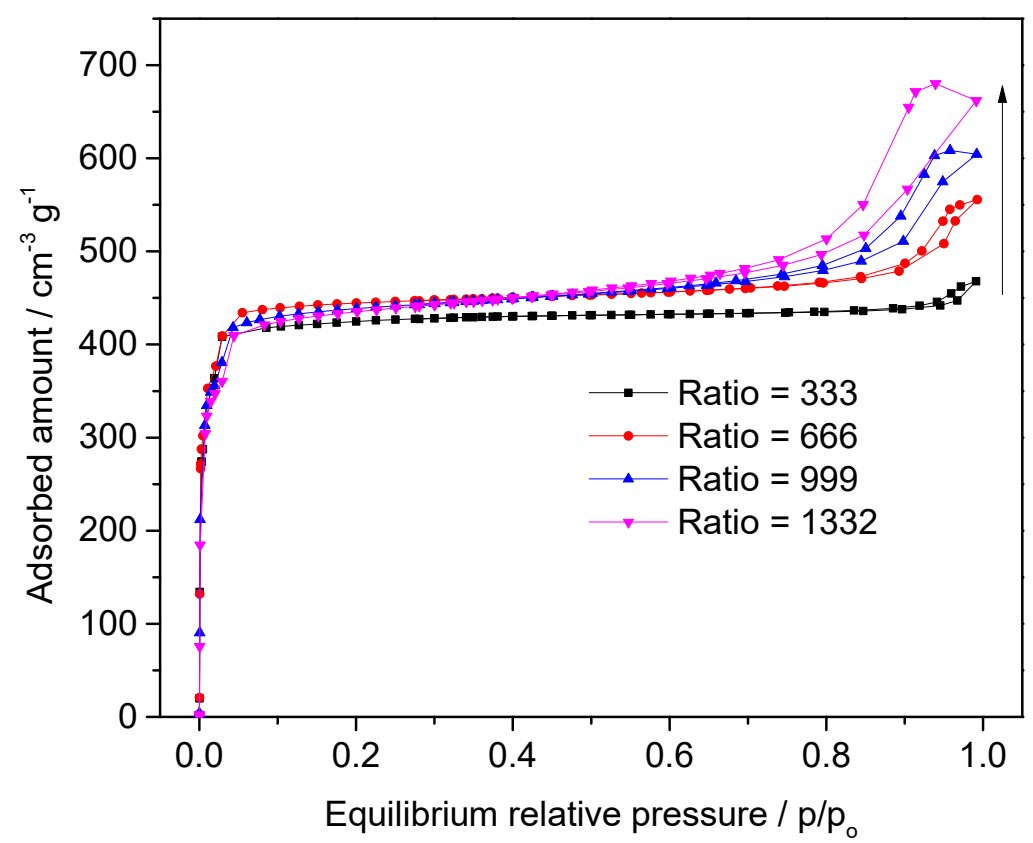

Figure 8. Sorption isotherms of nitrogen performed at $77 \mathrm{~K}$ on the ZIF-8 materials prepared with different dilution factors.

\subsection{Solvent nature influence}

In our study, we first decided to use pure methanol as solvent as some authors proved it appropriate. [28] Even being investigated longer than one decade, the synthesis of ZIF-8 is still interesting due to the influence of various parameters on the properties of the final products. Recently, Malekmohammadi et al. studied the impact of the solvent and ligand/metal molar ratio on the final characteristics of the synthesized ZIF-8 structures using conventional synthesis protocols at either room temperature or $130^{\circ} \mathrm{C}$.[29] They stated that methanol solvent easily resulted in pure phase of ZIF-8 while mixture of $\mathrm{MeOH} /$ water led to pure structure of ZIF-8 only at room temperature and ligand/metal molar ratio of 4 .

Zhang et al. followed four protocols reported from recent literature to obtained four samples of ZIF-8 with different crystallinity and studied their stability in water at room temperature.[30] They found that all samples were not stable, got dissolved in water to 
form zinc and imidazolate ions. The residual solid collected by filtration remained as ZIF-
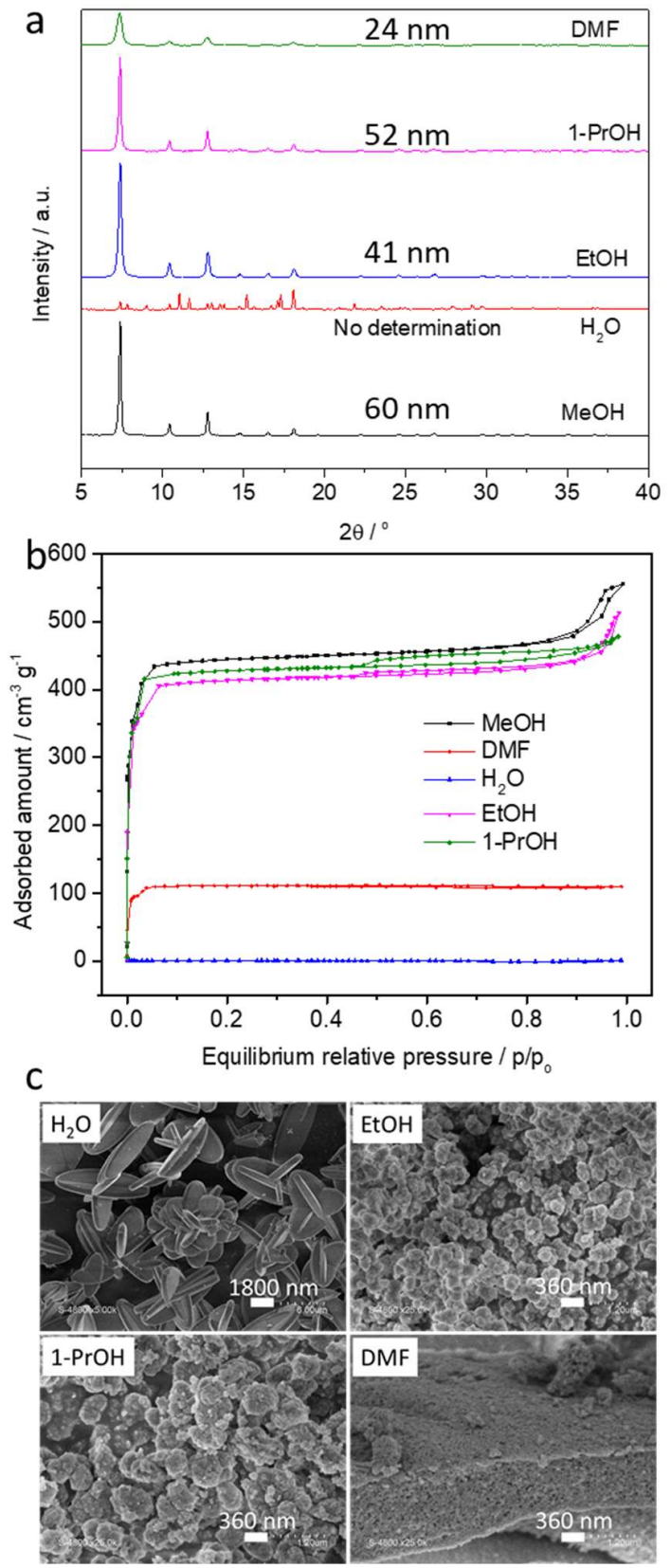

Figure 9. (a) XRD patterns of ZIF-8 prepared in different solvents, (b) Nitrogen sorption isotherms, (c) SEM images of ZIF-8 prepared in a) water, b) Ethanol, c) 1-Propanol, and d) DMF.
8 but the ones recovered by evaporation at $40^{\circ} \mathrm{C}$ forms a new phase of a crystalline structure with a dense, leaf-like morphology.

Using our optimized parameters, we varied the nature of solvent to confirm these results. Our results are presented in Figure 9. They are in line with the results published by Bustamante et al.[28] Aliphatic alcohols allows the formation of ZIF-8 nanoparticles as can be seen by XRD, SEM and further confirmed by nitrogen adsorption. Indeed, the XRD patterns exhibit the peaks usually observed for this material whereas type I sorption isotherms are found in these three cases. In the case of DMF, the peaks observed in the XRD pattern are broadened and they are not very intense. The nitrogen amount adsorbed at saturation is rather poor which suggests that the porosity of the material prepared using DMF as solvent is disrupted. In contrast, when water is used under the same synthesis conditions, the ZIF-8 phase is not obtained at all.

\subsection{Drugs encapsulation in ZIF-8 nanoparticles.}

Kaur et al. used one-pot synthesis to trap 6mercaptopurine into cages of ZIF-8 prepared by conventional stirring for $5 \mathrm{~min}$ in the presence of DMSO.[31] They found that the presence of drug 6mercaptopurine resulted in an increase of particle size compared to the bare ZIF-8 (200 nm vs $80 \mathrm{~nm}$ ). In addition, they also reported the presence of 
unknown impurity phase identified from XRD patterns.

Zheng et al. used a one-pot synthesis to encapsulate the anticancer drug doxorubicin (DOX) using the conventional stirring method with $\mathrm{H}_{2} \mathrm{O}$ as solvent which is questioning, according to our findings on the difficulty to prepare ZIF-8 using water as solvent.[17] Although they did not mention about any impurity, the XRD patterns were close to the one reported by Kaur et al.[31] They also found that the presence of drug DOX led to an increase of the particle size compared to pristine ZIF-8 $(100 \pm 30 \rightarrow 230 \pm 150 \mathrm{~nm})$. Other recent works related the use of ZIF-8 as carrier for drug delivery. For instance, Liedana successfully encapsulated caffeine in ZIF-8 nanoparticles, with loadings as high as $35 \mathrm{wt}$ $\%$. [32] It can be thought that a part of such a high amount is located on the external surface of ZIF-8 nanoparticles as crystalline clusters of drug. In our case, we focused on small amounts loaded, up to $5 \mathrm{~mol} \%$, to avoid any side crystalline formation of drugs in the synthesis medium. In Figure 10, the thermal analyses of the different materials are presented.
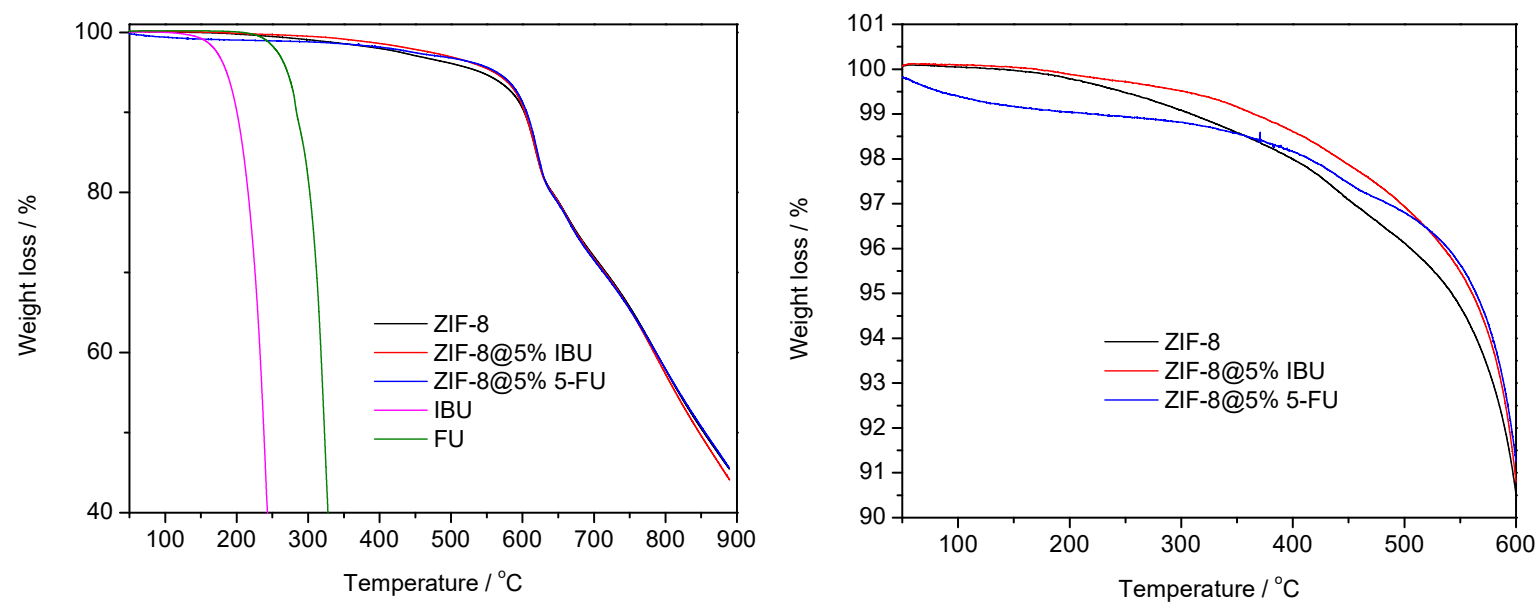

Figure10. Thermal analyses of the different materials performed under nitrogen; (left) full TGA curves, (right) inset at low temperature.

When focusing on the pure drugs, the weight losses observed are consistent with the Ibuprofen vaporizing temperature, $157^{\circ} \mathrm{C}$ and the 5 -FU decomposition temperature which is $283^{\circ} \mathrm{C}$. When comparing the TGA traces drugs@ZIF-8 nanoparticles with that obtained with pure ZIF-8, different traces are observed. This is the confirmation that drugs are 
included in ZIF-8. 5-Fluorouracil being hydrophilic, a water loss can be observed up to $150^{\circ} \mathrm{C}$. This can be better observed in Figure 10 right, where an inset of Figure 10 left is displayed. This weight loss does not exist in the case of pure ZIF-8, known to be hydrophobic, but also in the case of ibuprofen@ZIF-8, as ibuprofen is also known to be hydrophobic. The presence of drugs in the ZIF-8 cavities can also be deduced from the weight losses observed at higher temperatures. In the case of 5-FU, a clear sub-step can be distinguished between 400 and $45^{\circ} \mathrm{C}$. Its extent is $\sim 3.5 \mathrm{wt} \%$, which is consistent with the nominal insertion of $5 \mathrm{~mol} \%$ of $5-\mathrm{FU}$ in the material. Comparing pure $5-\mathrm{FU}$ with the encapsulated 5-FU in terms of stability, it can be noticed that the encapsulation plays a stabilizing role towards the decomposition of the encapsulated species. Indeed, the decomposition commences at $100^{\circ} \mathrm{C}$ higher in the case of 5-FU@ZIF-8 nanoparticles.

Focusing on ibuprofen@ZIF-8 nanoparticles, it can be noted that the trace is very close to that of pure $\mathrm{ZIF}-8$ at low temperature (below $200^{\circ} \mathrm{C}$ ). At higher temperature, ibuprofen@ZIF-8 is more stable, likely due to the reciprocal stabilization between ibuprofen and ZIF-8 which could originate from the pi-stacking between host and guest species. It has been shown in other systems.[33] The ibuprofen weight loss is more complex to define. By comparison with the trace obtained with pure ZIF-8, it can be deduced that it happens between $250^{\circ} \mathrm{C}$ and $300^{\circ} \mathrm{C}$, that is where the traces are not parallel, suggesting the occurrence of different decomposition processes.

In Figure 11, the XRD patterns of the materials prepared including ibuprofen and 5-FU drugs in the synthesis medium are shown. When comparing with the XRD pattern obtained with pure ZIF-8, similar patterns were obtained in the case of drugs@ZIF-8. The same number of peaks were obtained and the relative peaks intensities are also identical. The only difference is the cell parameter which has increased when drugs were included in the ZIF-8 structure. More precisely, the cell parameter varied from $a=16.981 \AA$ up to $a=16.992 \AA$ when 2.5 mol \% ibuprofen was included in the structure of ZIF-8. 


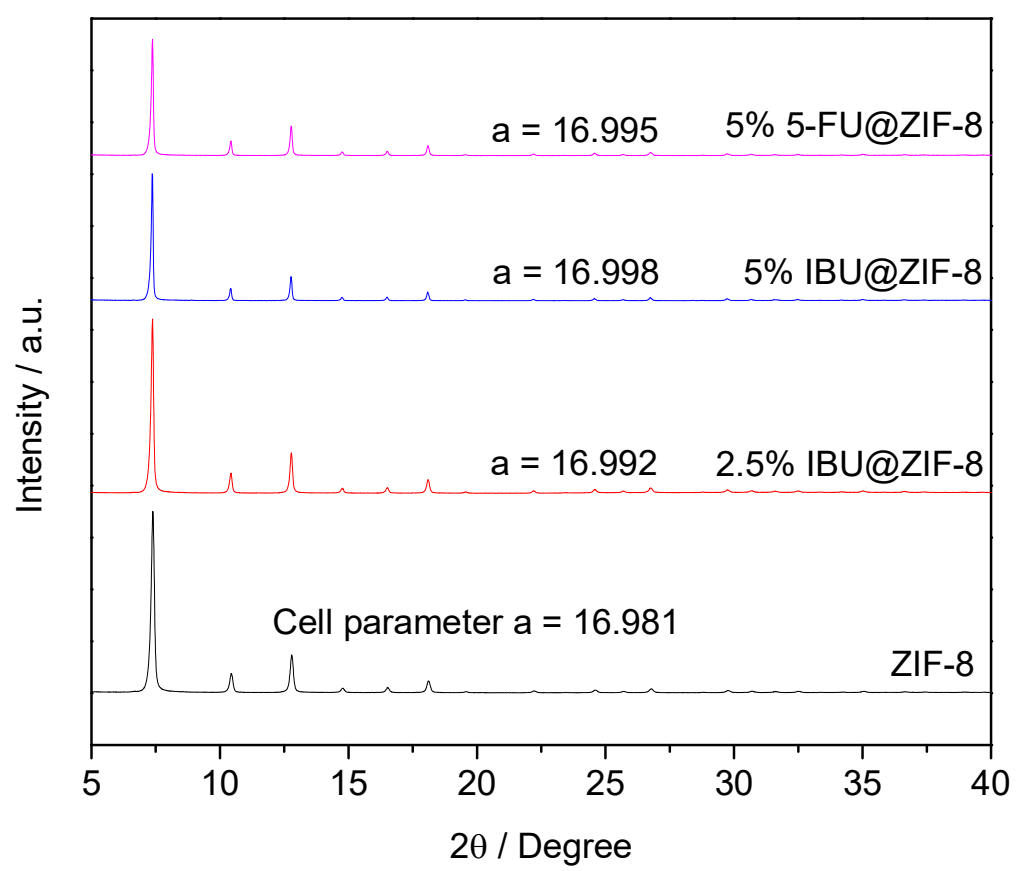

Figure 11. XRD patterns of ZIF-8 and drugs@ZIF-8 materials.

This suggests that to accommodate guests in its cavity, ZIF-8 suffered a slight enlargement of its cell dimensions. Increasing the proportion of drugs, up to $5 \mathrm{~mol} \%$, the cell parameters are further increased up to $16.998 \AA$ which is consistent with our former findings. Additionally, no extra peaks can be evidenced in the XRD patterns, confirming 
that the drugs were introduced as molecular entities, rather than agglomerated in the cavities but also on the external surface of ZIF-8. [34]
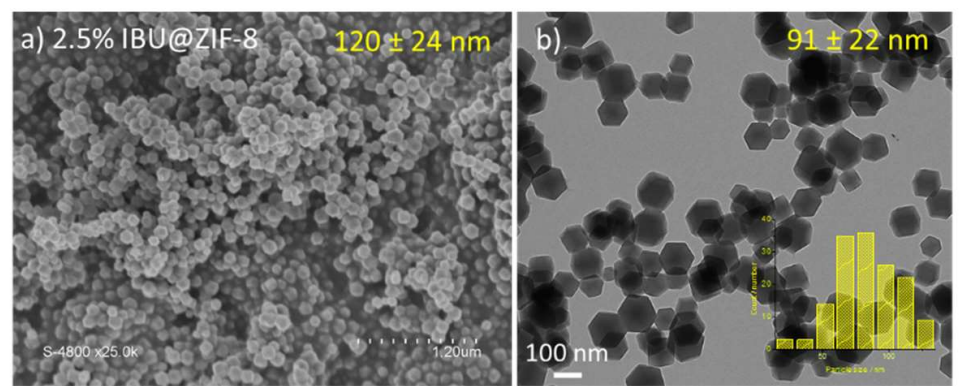

c) $5 \%$ IBU@ZIF-8 $150 \pm 30 \mathrm{~nm}$


Figure 12. Electron microscopies for the drugs@ZIF-8 nanoparticles (left) SEM; (right) TEM.
The influence of the encapsulation of drugs in ZIF-6 can be also seen by electron microscopies. In Figure 12, we gathered the obtained pictures corresponding to the different materials. It can be noted that the inclusions of drugs lead to very homogeneous hexagonalshaped nano-particles. Interestingly, the mean particle size increased, compared to pure ZIF-8 nanoparticles. This is in good accordance with former studies. [17,31] Additionally, increasing the amount of ibuprofen in ZIF-8 nanoparticles increased the particle size of the corresponding material.

Another evidence of the inclusion of drugs in ZIF-8 nanoparticles can be discussed, based on the nitrogen sorption isotherms. They have been plotted in Figure 13. As the amount of drugs encapsulated is moderate, their influence is rather on the sorption isotherms. It can be noted that these sorption isotherms belong to the type I, which indicates that the microporosity of the original ZIF-8 material is preserved to a large extent. The specific surface area of the different materials are reported in Table 1. A clear decrease can be noticed and it can be related to the amount of drug encapsulated in the materials. Indeed, the specific surface areas are reported as per gram of material which includes the weight of drug. The 
difference between the specific surface area of pure ZIF-8 and 5\% ibuprofen@ZIF-8 is close to $5 \%$ which corresponds to the weight of ibuprofen introduced. It can therefore be concluded that ibuprofen and 5-FU could be encapsulated in ZIF-8 nanoparticles, while preserving the microporosity, accessible to other processes, such as solvents, or simulated body fluids.

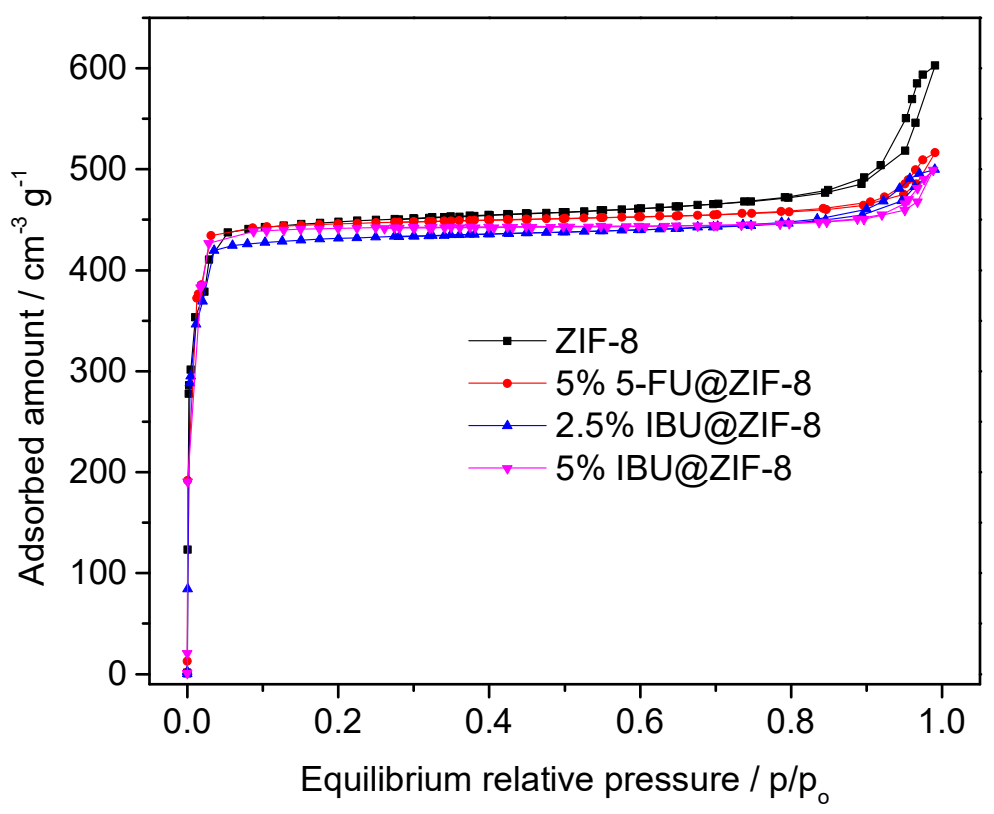

Figure 13. Nitrogen sorption isotherms performed on the drugs@ZIF-8 nanoparticles.

Table 1. Textural properties of the drugs@ZIF-8 materials

\begin{tabular}{llll}
\hline Material & $\mathrm{S}_{\mathrm{BET}} / \mathrm{m}^{2} \mathrm{~g}^{-1}$ & $V_{\text {micro }} / \mathrm{cm}^{3} \mathrm{~g}^{-1}$ & $\mathrm{~V}_{\text {total }} / \mathrm{cm}^{3} \mathrm{~g}^{-1}$ \\
\hline ZIF-8 & 1832 & 0.654 & 0.839 \\
$2.5 \%$ IBU@ZIF-8 & 1754 & 0.638 & 0.727 \\
$5 \%$ IBU@ZIF-8 & 1708 & 0.669 & 0.670 \\
$5 \%$ IBU@ZIF-8 & 1743 & 0.667 & 0.676 \\
\hline
\end{tabular}

\section{Conclusion.}

In this study, we first assessed the synthesis of ZIF-8 nanoparticles by evaluating the influence of key parameters, such as stirring, reaction time, solvent or dilution. It was shown that using ultrasound, the reaction time was highly reduced while preserving the 
structural and textural properties of ZIF-8 nanoparticles. It was also shown that adjusting the dilution ratio could be a simple way of reducing the mean particle size, which is essential for in vivo applications. Using the optimized parameters defined in this paper, we achieved the one-pot synthesis of ibuprofen@ZIF-8 and 5-FU@ZIF-8 nanoparticles. We ensured that adding the drugs to the reaction medium did not deny our former conclusions. Even though the particle size was enlarged when including the drugs, the nanoparticles thus prepared are in the nanosize domain. We are currently performing drug release in water and simulated body fluids with the same materials, before moving to other pharmaceuticals of interest.

Acknowledgments. We wish to thank T. Cacciaguerra and C. Biolley for performing Electron microscopy and TGA measurements.

\section{References}

[1] H. Furukawa, K.E. Cordova, M. O'Keeffe, O.M. Yaghi, The chemistry and applications of metal-organic frameworks, Science (80-. ). 341 (2013). doi:10.1126/science.1230444.

[2] P. Horcajada, R. Gref, T. Baati, P.K. Allan, G. Maurin, P. Couvreur, G. Férey, R.E. Morris, C. Serre, Metal-Organic Frameworks in Biomedicine, Chem. Rev. 112 (2012) 1232-1268.

[3] P. Horcajada, C. Serre, M. Vallet-Regí, M. Sebban, F. Taulelle, G. Férey, Metal-organic frameworks as efficient materials for drug delivery, Angew. Chem. Int. Ed. Engl. 45 (2006) 5874-5878.

[4] G. Lan, K. Ni, W. Lin, Nanoscale metal-organic frameworks for phototherapy of cancer, Coord. Chem. Rev. 379 (2019) 65-81. doi:10.1016/j.ccr.2017.09.007.

[5] T. Hidalgo, C. Serre, P. Horcajada, Nanostructured metal - organic frameworks and their bio-related applications, Coord. Chem. Rev. 307 (2016) 342-360.

[6] C. Doonan, R. Riccò, K. Liang, D. Bradshaw, P. Falcaro, MetalOrganic Frameworks at the biointerface: Synthetic Strategies and Applications, Acc. Chem. Res. 50 (2017) 1423-1432.

[7] M. Lismont, L. Dreesen, S. Wuttke, Metal-Organic Framework Nanoparticles in Photodynamic Therapy: Current Status and 
Perspectives, Adv. Funct. Mater. 27 (2017) 1-16.

doi:10.1002/adfm.201606314.

[8] P. Horcajada, T. Chalati, C. Serre, B. Gillet, C. Sebrie, T. Baati, J.F. Eubank, D. Heurtaux, P. Clayette, C. Kreuz, J.S. Chang, Y.K. Hwang, V. Marsaud, P.N. Bories, L. Cynober, S. Gil, G. Férey, P. Couvreur, R. Gref, Porous metal-organic-framework nanoscale carriers as a potential platform for drug deliveryand imaging, Nat. Mater. 9 (2010) 172-178. doi:10.1038/nmat2608.

[9] S. Rojas, T. Devic, P. Horcajada, Metal organic frameworks based on bioactive components, J. Mater. Chem. B. 5 (2017) 2560-2573.

[10] Q. Wu, M. Niu, X. Chen, L. Tan, C. Fu, X. Ren, J. Ren, L. Li, K. Xu, H. Zhong, X. Meng, Biocompatible and biodegradable zeolitic imidazolate framework/polydopamine nanocarriers for dual stimulus triggered tumor thermo-chemotherapy, Biomaterials. 162 (2018) 132143. doi:10.1016/j.biomaterials.2018.02.022.

[11] M. Hoop, C.F. Walde, R. Riccò, F. Mushtaq, A. Terzopoulou, X.Z. Chen, A.J. deMello, C.J. Doonan, P. Falcaro, B.J. Nelson, J. Puigmartí-Luis, S. Pané, Biocompatibility characteristics of the metal organic framework ZIF-8 for therapeutical applications, Appl. Mater. Today. 11 (2018) 13-21. doi:10.1016/j.apmt.2017.12.014.

[12] F.-M. Zhang, H. Dong, X. Zhang, X.-J. Sun, M. Liu, D.-D. Yang, X. Liu, J.-Z. Wei, Postsynthetic Modification of ZIF-90 for Potential Targeted Codelivery of Two Anticancer Drugs, ACS Appl. Mater. Interfaces. 9 (2017) 27332-27337.

[13] B. Chen, Z. Yang, Y. Zhu, Y. Xia, Zeolitic imidazolate framework materials: Recent progress in synthesis and applications, J. Mater. Chem. A. 2 (2014) 16811-16831. doi:10.1039/c4ta02984d.

[14] I. Abánades Lázaro, R.S. Forgan, Application of zirconium MOFs in drug delivery and biomedicine, Coord. Chem. Rev. 380 (2019) 230259. doi:10.1016/j.ccr.2018.09.009.

[15] J.B. James, Y.S. Lin, Kinetics of ZIF-8 Thermal Decomposition in Inert, Oxidizing, and Reducing Environments, J. Phys. Chem. C. 120 (2016) 14015-14026. doi:10.1021/acs.jpcc.6b01208.

[16] A. Tiwari, A. Singh, N. Garg, J.K. Randhawa, Curcumin encapsulated zeolitic imidazolate frameworks as stimuli responsive drug delivery system and their interaction with biomimetic environment, Sci. Rep. 7 
(2017) Article number: 12598.

[17] H. Zheng, Y. Zhang, L. Liu, W. Wan, P. Guo, A.M. Nyström, X. Zou, One-pot Synthesis of Metal-Organic Frameworks with Encapsulated Target Molecules and Their Applications for Controlled Drug Delivery, J. Am. Chem. Soc. 138 (2016) 962-968. doi:10.1021/jacs.5b11720.

[18] Z. Wang, X. Tang, X. Wang, D. Yang, C. Yang, Y. Lou, J. Chen, N. $\mathrm{He}$, Near-infrared light-induced dissociation of zeolitic imidazole framework-8 (ZIF-8) with encapsulated CuS nanoparticles and their application as a therapeutic nanoplatform, Chem. Commun. 52 (2016) 12210-12213. doi:10.1039/c6cc06616j.

[19] R. Raliya, C.T. Singh, K. Haddad, P. Biswas, Perspective on Nanoparticle Technology for Biomedical Use, Curr Pharm Des. 22 (2016) 2481-2490.

[20] N. Stock, S. Biswas, Synthesis of Metal-Organic Frameworks (MOFs): Routes to Various MOF Topologies, Morphologies, and Composites, Chem. Rev. 112 (2012) 933-969. doi:10.1021/cr200304e.

[21] S.R. Venna, J.B. Jasinski, M.A. Carreon, Structural Evolution of Zeolitic Imidazolate Framework-8 Surendar, JACS Commun. 132 (2010) 18030-18033. doi:10.1021/ja109268m.

[22] J. Zhuang, C.H. Kuo, L.Y. Chou, D.Y. Liu, E. Weerapana, C.K. Tsung, Optimized metal-organic-framework nanospheres for drug delivery: Evaluation of small-molecule encapsulation, ACS Nano. 8 (2014) 2812-2819. doi:10.1021/nn406590q.

[23] J. Cravillon, R. Nayuk, S. Springer, A. Feldhoff, K. Huber, M. Wiebcke, Controlling zeolitic imidazolate framework nano- and microcrystal formation: Insight into crystal growth by time-resolved in situ static light scattering, Chem. Mater. 23 (2011) 2130-2141. doi:10.1021/cm103571y.

[24] Y.R. Lee, M.S. Jang, H.Y. Cho, H.J. Kwon, S. Kim, W.S. Ahn, ZIF-8: A comparison of synthesis methods, Chem. Eng. J. 271 (2015) 276280. doi:10.1016/j.cej.2015.02.094.

[25] M.-S. Jang, H.-J. Kwon, W.-S. Ahn, Y.-R. Lee, H.-Y. Cho, S. Kim, ZIF8: A comparison of synthesis methods, Chem. Eng. J. 271 (2015) 276-280. doi:10.1016/j.cej.2015.02.094.

[26] K.S. Park, Z. Ni, A.P. Cote, J.Y. Choi, R. Huang, F.J. Uribe-Romo, H.K. Chae, M. O'Keeffe, O.M. Yaghi, ZIFs - first synthesis, Proc. Natl. 
Acad. Sci. U. S. A. 103 (2006) 10186-10191.

doi:10.1073/pnas.0602439103.

[27] T.D. Bennett, P.J. Saines, D.A. Keen, J.C. Tan, A.K. Cheetham, Ballmilling-induced amorphization of zeolitic imidazolate frameworks (ZIFs) for the irreversible trapping of iodine, Chem. - A Eur. J. 19 (2013) 7049-7055. doi:10.1002/chem.201300216.

[28] E.L. Bustamante, J.L. Fernández, J.M. Zamaro, Influence of the solvent in the synthesis of zeolitic imidazolate framework-8 (ZIF-8) nanocrystals at room temperature, J. Colloid Interface Sci. 424 (2014) 37-43. doi:10.1016/j.jcis.2014.03.014.

[29] M. Malekmohammadi, S. Fatemi, M. Razavian, A. Nouralishahi, A comparative study on ZIF-8 synthesis in aqueous and methanolic solutions: Effect of temperature and ligand content, Solid State Sci. 91 (2019) 108-112. doi:10.1016/j.solidstatesciences.2019.03.022.

[30] H. Zhang, M. Zhao, Y.S. Lin, Stability of ZIF-8 in water under ambient conditions, Microporous Mesoporous Mater. 279 (2019) 201-210. doi:10.1016/j.micromeso.2018.12.035.

[31] H. Kaur, G.C. Mohanta, V. Gupta, D. Kukkar, S. Tyagi, Synthesis and characterization of ZIF-8 nanoparticles for controlled release of 6mercaptopurine drug, J. Drug Deliv. Sci. Technol. 41 (2017) 106-112. doi:10.1016/j.jddst.2017.07.004.

[32] N. Liédana, A. Galve, C. Rubio, C. Téllez, J. Coronas, CAF@ZIF-8: One-step encapsulation of caffeine in MOF, ACS Appl. Mater. Interfaces. 4 (2012) 5016-5021. doi:10.1021/am301365h.

[33] A. Planchais, S. Devautour-Vinot, S. Giret, F. Salles, P. Trens, A. Fateeva, T. Devic, P. Yot, C. Serre, N. Ramsahye, G. Maurin, Adsorption of benzene in the cation-containing MOFs MIL-141, J. Phys. Chem. C. 117 (2013) 19393-19401. doi:10.1021/jp404474h.

[34] L. Boudjema, G. Toquer, A.H. Basta, P. Trens, D.A. Lerner, Confinement-Induced Electronic Excitation Limitation of Anthracene: The Restriction of Intramolecular Vibrations, J. Phys. Chem. C. (2018). doi:10.1021/acs.jpcc.8b09904. 
Graphical abstract :
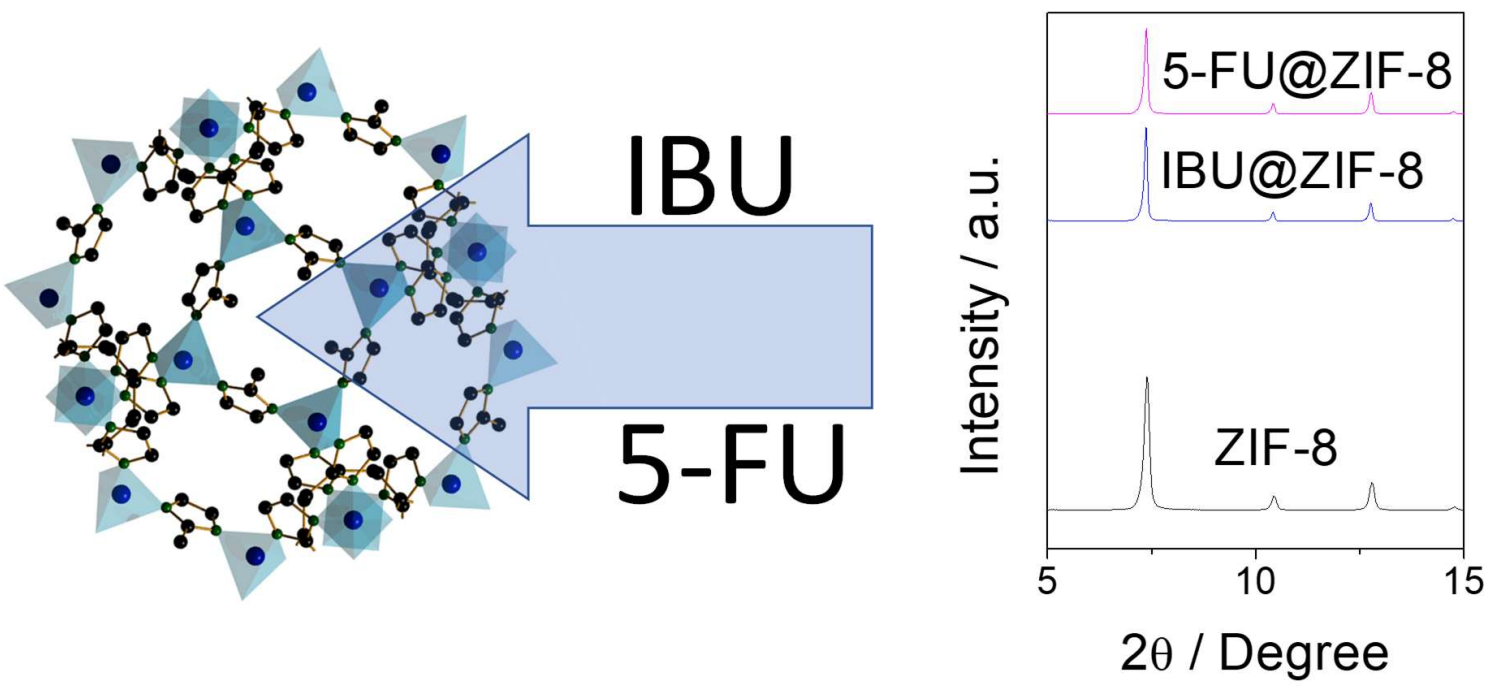